\title{
Yield drag in a two-dimensional foam flow around a circular obstacle: Effect of liquid fraction
}

\author{
Christophe Raufaste, ${ }^{1, *}$ Benjamin Dollet, ${ }^{1}$ 丹 Simon Cox,${ }^{2}$ Yi Jiang, ${ }^{3}$ and François Graner ${ }^{1}$ \\ ${ }^{1}$ Laboratoire de Spectrométrie Physique, BP 87, 38402 St Martin d'Hères Cedex, Franc $\AA^{\ddagger}$ \\ ${ }^{2}$ Institute of Mathematical and Physical Sciences, \\ University of Wales Aberystwyth, SY23 3BZ, UK \\ ${ }^{3}$ Theoretical Division, Los Alamos National Laboratory, Los Alamos, NM 87545, USA
}

(Dated: October 3, 2018)

\begin{abstract}
We study the two-dimensional flow of foams around a circular obstacle within a long channel. In experiments, we confine the foam between liquid and glass surfaces. In simulations, we use a deterministic software, the Surface Evolver, for bubble details and a stochastic one, the extended Potts model, for statistics. We adopt a coherent definition of liquid fraction for all studied systems. We vary it in both experiments and simulations, and determine the yield drag of the foam, that is, the force exerted on the obstacle by the foam flowing at very low velocity. We find that the yield drag is linear over a large range of the ratio of obstacle to bubble size, and is independent of the channel width over a large range. Decreasing the liquid fraction, however, strongly increases the yield drag; we discuss and interpret this dependence.
\end{abstract}

\section{INTRODUCTION}

Multiphase materials such as colloids, emulsions, polymer or surfactant solutions, wet granular systems and suspensions of deformable objects like red blood cells are characterized by a complex mechanical behaviour [1], due to the interaction of their constitutive entities. The concentration is one of the key parameters which control the rheology, determining especially the transition from liquid-like to solid-like properties [2].

Amongst these complex fluids, liquid foams provide a convenient model experimental system for laboratory studies of the interplay between structure, concentration and rheology. This is because the bubbles which constitute the foam's internal structure can be easily visualised and manipulated. The mechanical behaviour of foams is very diverse: they appear elastic, plastic or viscous depending on the deformation and velocity

*Address for correspondence: craufast@ujf-grenoble.fr

${ }^{\dagger}$ present address: Physics of Fluids, University of Twente, The gradient [3, 4].

A liquid foam consists of gas bubbles separated by a connected network of liquid boundaries. This liquid phase occupies a fraction $\Phi$ of the volume of the foam. The "dry foam" limit, in which $\Phi$ tends to zero, corresponds to polyhedral bubbles separated by thin walls. It is associated with a divergence of certain contributions to the viscous dissipation [5]. However, the foam's nondissipative properties (such as surface energy 6], shear modulus or yield stress [7, 8]) usually tend to a regular, finite limit when the liquid fraction $\Phi$ tends to zero.

The total "yield drag" $F_{Y}^{t}$ is the minimal force observed when there exists (or, equivalently, required to create) a movement of the foam relative to an obstacle [9]. It is a global, geometry-dependent quantity directly measurable in experiments and in practical applications of foams, for instance when a foam flows through a porous medium [10], or when one introduces an object into a foam (analogous to sticking one's finger into shaving cream).

In the low-velocity limit (in which viscous dissipation is neglected 11,12 ) the total yield drag $F_{Y}^{t}$ has two 
bubbles, denoted $F_{Y}^{p}$, and the network of bubble walls (i.e., soap films with surface tension), $F_{Y}^{n}$. Thus

$$
F_{Y}^{t}=F_{Y}^{p}+F_{Y}^{n}
$$

Here we consider the network contribution $F_{Y}^{n}$ and show how it is affected by the liquid content of the foam.

We consider a single layer of equal-area bubbles to facilitate preparation and analysis of experiments, as well as numerical and analytical modelling [13]. Section $\Pi$ presents a 2D flow of a quasi-2D foam (a bubble monolayer) around a fixed circular obstacle within a long channel: this is the historical experiment of Stokes, already adapted to foams both in 2D 9, 14] and 3D 15, 16, 17, 18] flows. We compare them with truly 2D simulations using two physically equivalent but differently optimised software packages (Section III). The simulation methods allow easy variation of the geometrical parameters such as bubble, obstacle and channel size and better control of bubble area. In Section IV we discuss the issue of a common, unambiguous definition of liquid fraction for all systems, in theory, experiments and simulations. Section $\mathrm{V}$ presents our results: we show that the yield drag displays the expected dependence with the bubble, obstacle and channel size, and increases when the liquid fraction $\Phi$ decreases. The discussion in Section VI emphasises that taking into account the effect of liquid fraction allows all data to be plotted on a single master-curve and that, although they cover different ranges of $\Phi$, the results of both simulation and experiment are consistent with a simple model.

\section{EXPERIMENTAL METHODS}

\section{A. Foam channel}

Our bulk soap solution is de-ionised water with $1 \%$ Teepol, a commercial dish-washing liquid. Its surface tension, measured with the oscillating bubble method (that is, imaging the interface shape), is $\gamma=26.1 \pm 0.2$ $\mathrm{mN} \mathrm{m}^{-1}$, and its kinematic viscosity, measured with a
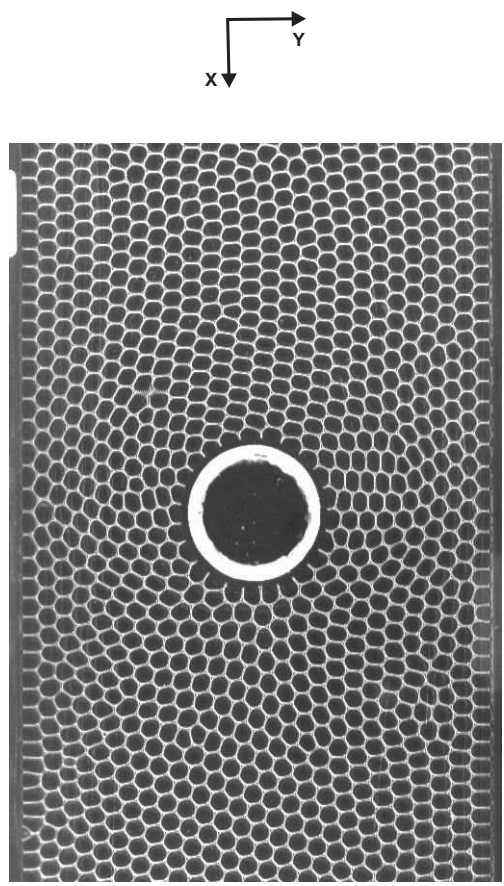

FIG. 1: Image of the experiment, with the foam confined between liquid and glass and flowing from top to bottom. Foam thickness $h=4.5 \mathrm{~mm}$; bubble area $A=16 \mathrm{~mm}^{2}$; obstacle diameter $d_{0}=3 \mathrm{~cm}$; mean velocity $v=5.6 \mathrm{~mm}$ $\mathrm{s}^{-1}$; effective liquid fraction $\Phi=0.06$.

The experimental set-up [9] confines the foam between a liquid reservoir and a glass lid ("liquid-glass" set-up [19]). A $1 \mathrm{~m}$ long, $w_{c}=10 \mathrm{~cm}$ wide tank is filled with soap solution, leaving below the glass lid a free space of thickness $h$ which we can adjust. We will call this parameter the "foam thickness" for simplicity. At its centre is a circular obstacle of diameter $d_{0}=3$ (Fig. 1) or $4.8 \mathrm{~cm}$. At the entrance to the channel, nitrogen is blown at a computer-controlled flow rate, which varies between 5 and $500 \mathrm{ml} \mathrm{min}^{-1}$. A typical value of the average velocity is $3 \mathrm{~mm} \mathrm{~s}^{-1}$, for a $3.5 \mathrm{~mm}$ thickness and a flow rate of $50 \mathrm{ml} \mathrm{min}{ }^{-1}$.

The resulting foam consists of a horizontal monolayer of bubbles. It exits freely at atmospheric pressure at the open end of the channel, $P=P_{a t m}$. In the absence of the obstacle, it yields a two-dimensional plug flow. With the obstacle present, the flow remains twodimensional (even though the foam itself is not exactly 
Due to the presence of the obstacle, there is a velocity gradient. There are many bubble rearrangements (so called "T1s" or neighbour-swapping events): two three-fold vertices contact, merge and re-separate. We observe well-separated T1s; that is, between two T1s, there is enough time for the foam to relax to an equilibrium state. The present flow is slow enough that we can extrapolate the results to the low velocity limit [9], where a comparison with quasi-static calculations and simulations makes sense [20]. (Although note that this is distinct from the zero-velocity case, that is the absence of flow.) The data presented below are all in this limit, extracted from the experiments as described in Appendix A 1

The bubble walls meet the solid boundaries of the foam (glass plate, lateral channel walls, obstacle itself) at a $90^{\circ}$ angle [21]. The surface density of bubbles is $1 / A$, where $A$ is the average area per bubble (including its walls). The foam is monodisperse: the area variation at the channel entrance is less than 5\%. The average area is fixed at a value ranging from $0.121 \mathrm{~cm}^{2}$ to 0.393 $\mathrm{cm}^{2}$; most experiments have $A=0.160 \mathrm{~cm}^{2}$. Despite the low velocity, and hence the long transit time, we detect neither bubble coalescence nor coarsening. The effect of foam ageing on rheology [22, 23] is thus negligible.

\section{B. Force measurements}

\section{Total yield drag}

The obstacle floats just below the top glass surface and is free to move, without solid friction. However, it is linked to a fixed base through a calibrated elastic fibre. We track the obstacle displacement from its position at rest using a CCD camera which images the foam flow from above. We thus measure the force exerted by the flowing foam on the obstacle (precision better than 0.1 $\mathrm{mN})$ [9].

We check that the lift (spanwise component of the as expected by symmetry (data not shown). After a transient, the total drag $F^{t}$ (streamwise component of the resultant force) fluctuates around a steady value: we record the average and standard deviation of these steady flow data. The extrapolation to the low velocity limit (or zero-velocity intercept) of the force-velocity curve defines the yield $\operatorname{drag} F_{Y}^{t}$. It is independent of the bulk solution viscosity [24], and increases with the obstacle to bubble size ratio [9].

In this paper, we reanalyse the data already published in [9] at various bubble areas, and we present new data for another control parameter: the foam thickness. These data are presented in Appendix A 1 As explained in Sec. IVB the foam thickness provides a means by which to vary the liquid fraction in experiments.

\section{Network contribution to the yield drag}

We measure $F_{Y}^{n}$ as follows. Each bubble wall in contact with the obstacle pulls it with a force equal to its line tension $\lambda$ (the energy per unit length, which is of order $2 \gamma h$, see Appendix A 2 . The elastic contribution of the wall network to the drag is then the vectorial sum of all these individual forces, which all have the same modulus $\lambda$. As mentioned above, in a quasi-static flow each wall touches the obstacle at $90^{\circ}$ angle. Thus it suffices to find the contact points between bubble walls and the obstacle and sum all outward normal vectors to the obstacle vectorially at these contact points (which is easy to determine for a circular obstacle). If the downstream geometry of the foam was the same as that upstream, the drag would be zero. Since bubbles are squashed upstream and stretched downstream, the asymmetry means that there are more bubble walls pulling the obstacle downstream, and we measure a downstream elastic contribution to the drag, $F_{Y}^{n} / \lambda$.

The actual value of the line tension $\lambda$ is unimportant in what follows, where only measurements of $F_{Y}^{n} / \lambda$ are compared. However, as presented in detail in Appendix 
of magnitudes of the independent measurements of $F_{Y}^{n}$ and $F_{Y}^{t}$.

\section{SIMULATIONS}

\section{A. Deterministic simulations (the Surface Evolver)}

The Surface Evolver [25, 26] offers the possibility to reach a true quasi-static limit, that is a succession of exact equilibrium states, through a deterministic minimisation of the foam's energy. It yields precise details of the foam structure.

\section{Preparation of the foam}

We use a mode in which all bubble walls are represented as circular arcs. The Surface Evolver lets these circular arcs evolve in order to minimise the total perimeter (equivalent to the energy, up to the prefactor $\lambda$ ). It enforces the constraint that bubble areas $A$ remain fixed and determines the corresponding Lagrange multipliers, namely each bubble's pressure $P$. Since we can freely choose the units, we call them "cm" and use bubble size $A=0.16$ or $0.353 \mathrm{~cm}^{2}$, channel width $w_{c}=10 \mathrm{~cm}$, and obstacle diameters $d_{0}=1.5,3$ and $4.8 \mathrm{~cm}$, to reproduce actual experiments.

The lateral sides of the channel are rigid and do not interact with the foam, ensuring free-slip boundary conditions for the flow, resulting in a $90^{\circ}$ angle where a bubble wall meets the side. We adopt a periodic boundary condition in the direction of motion: bubbles that exit at the end of the channel are fed back into the entrance of the channel. We stop the simulation when each bubble has passed the obstacle no more than once.

We begin with a rectangular lattice of $30 \times 25$ monodisperse bubbles of area slightly larger than the required area $A$. We randomly perturb this lattice so that all the unstable four-fold vertices dissociate into pairs of three-fold vertices and the whole foam struc- (a)
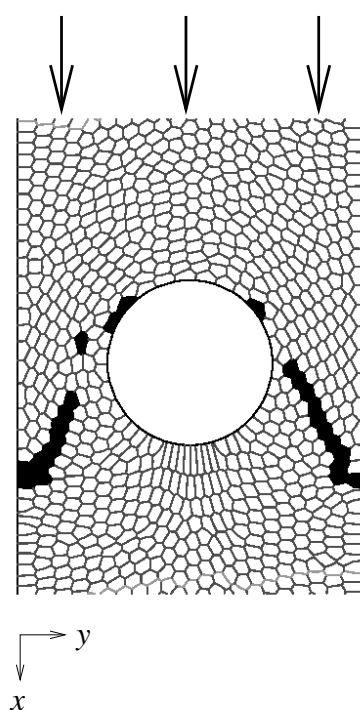

(b)

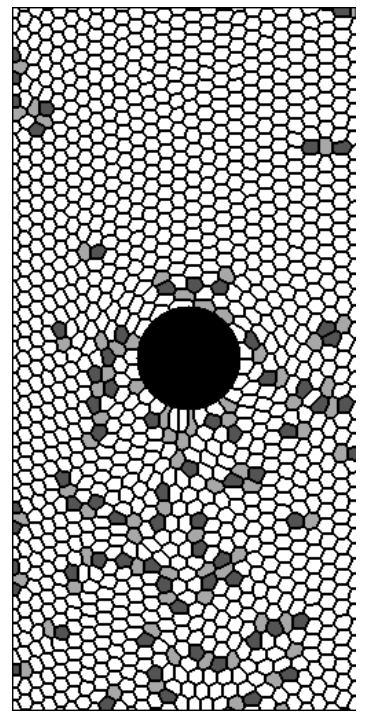

FIG. 2: Images of simulated foam flow. The $x$-axis is parallel to the flow along the channel, with periodic boundary conditions (exiting bubbles re-enter); axis $y$ is spanwise, with free-slip rigid boundary conditions on either side of the channel. (a) Surface Evolver. The image shows the whole simulation domain of 750 bubbles; the shaded bubbles started in a horizontal line. Here $d_{0}=4.8 \mathrm{~cm}, A=0.16$ $\mathrm{cm}^{2}, w_{c}=10 \mathrm{~cm}, L_{c}=0.05 \mathrm{~cm}$ and therefore $\Phi=0.0037$. (b) Potts model. The image shows the simulated channel's full width (except for a few pixels) of 256 pix, and half its length. Here $d_{0}=74$ pix, $A=100 \mathrm{pix}^{2}$ and $\Phi=0.005$. Bubbles coloured in white are without topological defect: 6-sided bulk bubbles, or 5-sided bubbles touching a lateral wall or the obstacle [6]. Bubbles with fewer neighbours are 
bubble to be the circular obstacle, and slowly increase its area to the required value (and correspondingly reduce the bubble areas to $A$ ) and constrain its edges to lie on a circle. The centre of the circular obstacle is then moved to the centre of the channel and the structure again relaxed to equilibrium.

\section{Simulation of the flow}

With the obstacle in the desired location and the foam close to equilibrium, we start the quasi-static iteration procedure. This requires that we move the foam past the obstacle, in a direction which we denote by $x$; the difficulty is in doing this with the periodic boundary conditions without fixing any vertices or bubble shapes. Our method is to choose a continuous line of consecutive bubble walls from one side of the channel to the other. Joining this to a line at $x=0$ with lines along the channel walls defines a plane region with a certain area, which we constrain. At each iteration we choose a convenient line of consecutive walls, and increment the target area of the region formed by a small amount $d A$ (equal to $0.05 \mathrm{~cm}^{2}$ in all simulations), resulting in a slight movement of a line of films without modifications to the bubble areas. The total perimeter of the structure is then reduced until it converges to a constant value (Fig. 2a), so that measurements can be performed.

We have double-precision values for the network geometry. We measure the network contribution $F_{Y}^{n}$ to the yield drag as in experiments (section IIB2). It is the sum of the unit vectors of the bubble wall with one end attached to the obstacle, expressed in units of the line tension (hence as a dimensionless number). Here too, we check that the lift is consistently zero within fluctuations (data not shown).

With the area increment $d A=0.05 \mathrm{~cm}^{2}$, the transient lasts for about 600 iteration steps (Fig. 3a). This is comparable to, but still smaller than, the total simulation time that is reasonably accessible. After this tran-

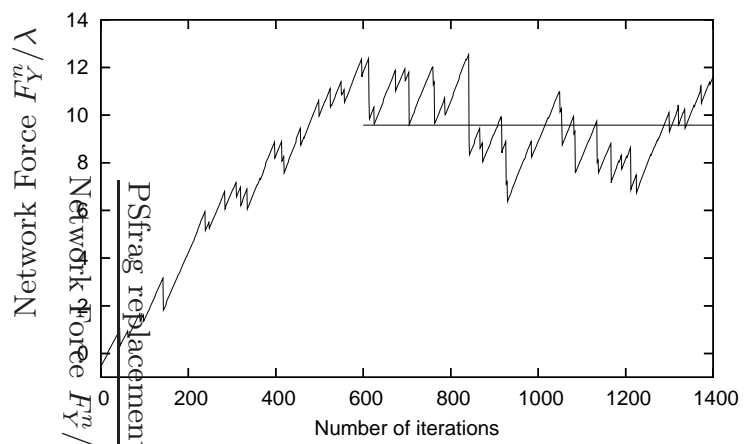

(a)

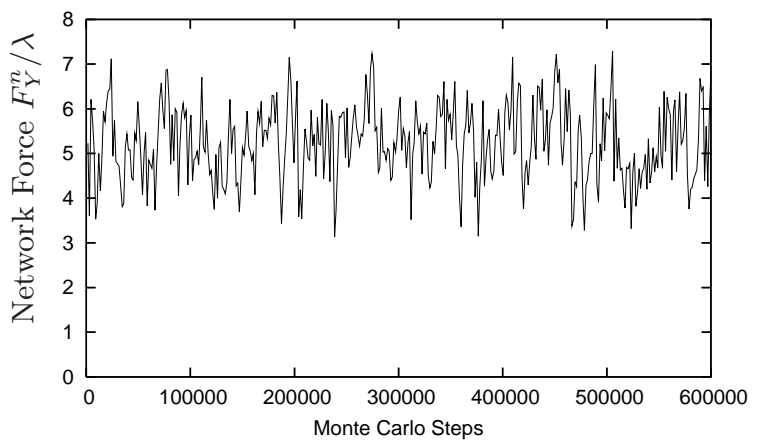

(b)

FIG. 3: The network force $F_{Y}^{n}$ (expressed in units of the line tension $\lambda$ ) measured in simulations versus time. (a) Surface Evolver data plotted every iteration step; $d_{0}=4.8$ $\mathrm{cm}, A=0.16 \mathrm{~cm}^{2}$ and $L_{c}=0.05 \mathrm{~cm}, \Phi=0.004$. The plateau value is $F_{Y}^{n}=9.6 \pm 1.4$. (b) Potts model data plotted every 1500 Monte Carlo Steps; $d_{0}=74$ pix, $A=100$ pix $^{2}$, $\Phi=0.005$. The plateau value is $F_{Y}^{n}=5.4 \pm 1.1$.

fluctuations, due to the rearrangements of the bubbles, recall the stress drops observed in Couette experiments for disordered foams [27, 28]. We record the average and standard deviation of these plateau (steady-flow) data for a total of $1500-600=900$ iterations. To validate the choice of our simulation size, we checked once that the drag forces are the same with more bubbles in the direction of flow (1250 bubbles instead of 750), although the transient is longer.

Each simulation takes about 35 hours on a Pentium IV 3.20 GHz processor: typically a several hour buildup to the initial structure (inflating the obstacle), plus one iteration per minute (depending on the number of 


\section{B. Stochastic simulations (Potts Model)}

To simulate a larger number of bubbles, the Potts model adapted for foam rheology [29] also minimises the same energy, but stochastically (Monte-Carlo), which increases the simulation speed. It thus provides more statistics on $F_{Y}^{n}$ and allows quicker variation of the geometrical parameters.

\section{Principle of the Potts Model}

The Potts model is derived from a large- $Q$ Potts model run at zero temperature, a model widely used to model grains in crystals 30]. It has been also applied to different domains of foam physics, including rheology, by enforcing the conservation of bubble size and applying an external force [29].

We consider a 2D square lattice. Each site $i$ has an integer index $\sigma_{i}$. The $k^{t h}$ bubble is defined as the domain consisting of all sites with the same index value $\sigma_{i}=k$. Thus bubbles tile the plane without gaps or overlaps. The evolution is driven by the minimisation of a total energy $\mathcal{H}$ (strictly speaking, it is a Hamiltonian), which has the same three physical ingredients as in the Surface Evolver: interfacial energy, area constraints, external forcing of the flow. Since the calculations are performed on a lattice, we have

$$
\begin{aligned}
\mathcal{H}= & \lambda \sum_{i, j \text { neighbours }}\left[1-\delta\left(\sigma_{i}, \sigma_{j}\right)\right] \\
& +\chi \sum_{\text {bubbles } k}\left(A_{k}-A_{k}^{t}\right)^{2}+b \sum_{\text {sites } i} x_{i} .
\end{aligned}
$$

The first term represents the contribution of the energy of the interfaces between the bubbles. Minimising this term leads to perimeter minimisation. Here $\delta$ is the Kronecker symbol: $1-\delta$ is equal to 1 if the neighbouring sites $i, j$ belong to different bubbles $\left(\sigma_{i} \neq \sigma_{j}\right)$; else it equals zero. We choose to evaluate this term with the fourth nearest neighbour interactions to obtain an isotropic line tension insensitive to the details of the lattice 31].

The second term keeps each bubble area $A_{k}$ (the num- target value $A_{k}^{t}$. Here $\chi$ is the compressibility, which we choose to be high enough to keep bubble areas constant to within a few pixels. The balance between this term and the preceding one simulates a foam relaxing towards mechanical equilibrium.

The third term is a bias term that describes an energy gradient, hence a homogeneous external force field. Here $b$ is the bias intensity and $x$ the site's coordinate along the flow. Without obstacle, the resulting velocity profile would be a plug flow.

We use a Metropolis algorithm to evolve the foam: we randomly select a site at a bubble boundary, change its index to the value of a neighbour if and only if this decreases the total energy (eq. 2). Several independent changes are tried successively; a Monte Carlo Step (MCS) is defined conventionally as a number of tries equal to the total number of lattice sites.

\section{Simulation of the flow}

As for the Surface Evolver (section III A 1), we choose a periodic boundary condition in the direction of flow and free-slip rigid boundary conditions on the channel sides. To ensure that it does not affect the steady-state measurements presented below, the total channel length is $4 w_{c}$, out of which only $2 w_{c}$ are used for measurements and are shown on Fig. (2b).

To match the experiments, we choose $16 \leq d_{0} \leq 148$ pix, $64 \leq A \leq 400$ pix $^{2}$ and $64<w_{c}<512$ pix. Initially, we insert a rigid round obstacle in the centre of the channel, and let a perfectly ordered foam (honeycomb pattern) flow in. We then switch off the bias term by setting $b=0$, and relax the foam to ensure that the bubbles recover their (near) equilibrium state. The foam has reached the stationary state at the end of this preparation.

We then switch the bias on again, and use the smallest bias $b$ for which the foam flows, which is constant and independent of parameters such as bubble diameter. We perform measurements at intervals of 1500 
We measure the network contribution to the drag using the same method as in the experiments and Surface Evolver simulations. It fluctuates around a steady value: we record the average and standard deviation of these plateau (steady-flow) data (Fig. 3b). We run each simulation for a total of 600,000 MCS, during which a bubble passes completely through the channel but no bubble passes the obstacle twice. One simulation takes about 12 hours on a Pentium IV $2.8 \mathrm{GHz}$ processor.

\section{LIQUID FRACTION}

In ideal 2D foams (Sec. IVA), given $A$, the liquid fraction scales as the square of the vertex radius. This radius in turn relates to a cut-off length $L_{c}$, that is the length at which a bubble edge becomes unstable and the surrounding bubbles undergo a T1. This length $L_{c}$ is always defined, and is relevant to the mechanical properties investigated here. In experiments, $L_{c}$ depends on the actual (3D) shape of bubbles; in simulations, $L_{c}$ is an input parameter.

It suggests a coherent definition of the effective liquid fraction, presented below, consistent within and between experiments, simulations and theory. Note that we consider here a foam dry enough to have a non-zero shear modulus [32], that is, below the critical liquid fraction 7] (see eq. 8 for the honeycomb value).

\section{A. Ideal 2D foams}

In an ideal 2D foam, a Plateau border is a triangle with concave edges of radius $R$ which match tangentially three straight lines meeting at $120^{\circ}$ (Fig. (4). The area $A_{P B}$ of a Plateau border is [3] :

$$
A_{P B}=\left(\sqrt{3}-\frac{\pi}{2}\right) R^{2} .
$$

The liquid fraction $\Phi$ is defined as:

$$
A_{l}=A \Phi,
$$

where $A_{l}$ is the area occupied by the liquid. Since each

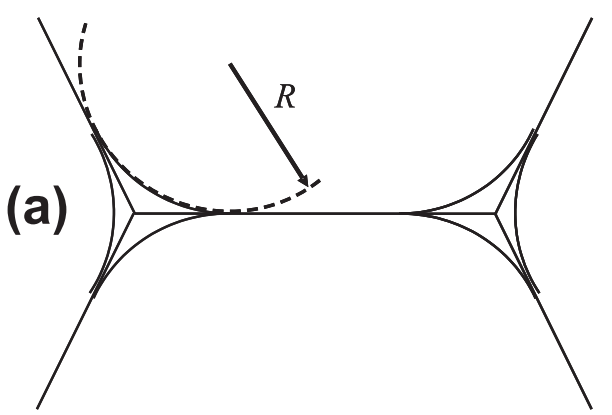

(b)

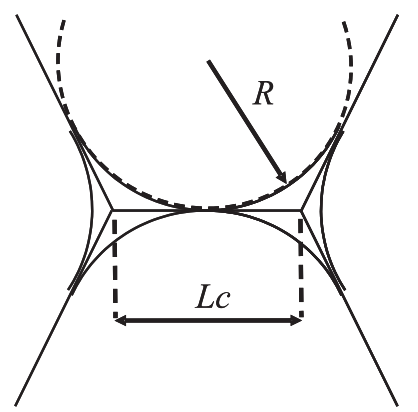

FIG. 4: Picture of two adjacent three-fold vertices with Plateau borders. (a) We apply the decoration theorem 32] to model a wet foam. The liquid is present only at the vertices, and (assuming here straight or nearly-straight walls) the uniformity of pressure $P$ inside bubbles forces each gas/liquid interface to have the same radius of curvature, $R$. (b) Critical position of the vertices just before the "T1" neighbour-swapping event. It defines the cut-off wall length $L_{c}$.

tween 3 bubbles, we have $A_{l}=n A_{B P} / 3$. For a honeycomb array of bubbles, $n=6$ and:

$$
\Phi=(2 \sqrt{3}-\pi) \frac{R^{2}}{A}
$$

Here we consider foams where bubbles have the same area (monodisperse foams), but not necessarily the same number of sides $n$ (topological disorder). Since on average over the whole foam $\bar{n} \approx 6[3]$, and since Plateau borders have almost the same size and radius of curvature, eq. (5) still holds here approximately.

A T1 is triggered when the distance between these vertices becomes smaller than a cut-off wall length $L_{c}$, which increases with $R$, and thus with $\Phi$. To make this observation more quantitative, one possible convention 
(Fig. 4):

$$
\frac{R}{\sqrt{3}}=\frac{L_{c}}{2},
$$

so that, together with eq. (5):

$$
\Phi=\frac{3}{2}\left(\sqrt{3}-\frac{\pi}{2}\right) \frac{L_{c}^{2}}{A} \approx 0.242 \frac{L_{c}^{2}}{A} .
$$

Given $A$, the physical information conveyed by $R, \Phi$ or $L_{c}$ is the same. For comparison between different experiments or simulations, we use $\Phi$ because it is dimensionless.

For an ideal honeycomb without shear, all vertices merge at the same liquid fraction: the hexagons become circular when $L_{c}$ equals the side-length of the hexagons; the bubbles are circular, with a radius equal to $R$. This critical liquid fraction is [7]:

$$
\Phi_{c}=1-\frac{\pi}{2 \sqrt{3}}=0.0931 .
$$

\section{B. Experiments}

In the experiments, the actual (3D) shape of bubbles is determined by the foam thickness. Fig. (1) shows a foam thickness of $4.5 \mathrm{~mm}$; beyond this thickness, the bubbles undergo a three-dimensional instability and the foam is no longer a monolayer [33]. At the other extreme, at $2 \mathrm{~mm}$ and below, the bubbles are circular and separated: both the foam's 2D shear modulus and the yield drag vanish. When $h$ increases, $L_{c}$ decreases, thus $\Phi$ decreases too (Fig. 9 in Appendix $\mathrm{A}$ ).

We show in Appendix B that there is a correspondence between $L_{c}$ and the length $L_{\max }$ of the edge of a bubble just attached to the obstacle (Fig. 11k in Appendix (B). Since $L_{\max }$ is much bigger than $L_{c}$, it can be estimated with much more precision in experiment (the uncertainty in both $L_{c}$ and $L_{\max }$ is one pixel).

We measure on the skeletonized image the length $L_{\max }$ several times preceding its disappearance during a $\mathrm{T} 1$ process, and keep the average as $L_{\max }$ and the stan-

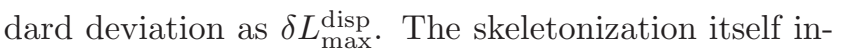
duces a systematic error in determining the actual posi- the systematic error on $L_{\max }$. The total uncertainty on $L_{\max }$ is therefore $\delta L_{\max }=\sqrt{\left(\delta L_{\max }^{\text {disp }}\right)^{2}+\left(\delta L_{\max }^{\text {skel }}\right)^{2}}$.

To deduce $\Phi$ from the measurements of $L_{\max }$, we combine Eqs. (5) and (B3) to get the following expression as a function of $L_{\max }^{2} / A$ only:

$$
\Phi=\frac{3}{2} \frac{2 \sqrt{3}-\pi}{2+\sqrt{3}}\left(\frac{\sqrt{A}}{L_{\max }}-\frac{L_{\max }}{4 \sqrt{3 A}}\right)^{2} .
$$

Its uncertainty is:

$$
\frac{\delta \Phi}{\Phi}=2 \frac{\delta L_{\max }}{L_{\max }} \frac{\sqrt{A} / L_{\max }+L_{\max } / 4 \sqrt{3 A}}{\sqrt{A} / L_{\max }-L_{\max } / 4 \sqrt{3 A}} .
$$

\section{Simulations}

The Surface Evolver requires that we specify explicitly the cut-off wall length $L_{c}$ at which two three-fold vertices are allowed to contact, merge and re-separate. Since $L_{c}$ is an input parameter, it is determined without uncertainty. This defines explicitly an effective liquid fraction (eq. 7), at least for small values of $\Phi$. We choose $L_{c}$ to be of the order of $0.1 \mathrm{~cm}$ or slightly smaller, reaching $\Phi=0.0015,0.0037,0.0061$ and 0.015 . At very small values of $\Phi<610^{-6}$, films behind the obstacle would get very stretched and lead to numerical problems. Attempting larger values of $\Phi>0.015$ would lead to poor convergence in the Surface Evolver and would require that we simulate the actual geometry of the liquid in the vertices (including 4-fold vertices).

In the Potts model, the cut-off distance $L_{c}$ at which two vertices merge is either 1 or 2 pixels. We use this range to define the uncertainty on the value of $\Phi$. Since we will plot the results in log scale, we choose $L_{c} \approx \sqrt{2}$ and $\Phi \approx 0.242 \times 2 / A \approx 0.5 A^{-1}$ to lie in the middle of this interval. Thus the area $A$ of bubbles (that is, the number of pixels per bubble) defines an effective liquid fraction, at least for small values of $\Phi$. The simulated range $64 \mathrm{pix}^{2} \leq A \leq 400 \mathrm{pix}^{2}$ corresponds to $0.00125<\Phi<0.0075$, large enough to describe realistically the shape of bubbles, and small enough to keep 


\section{RESULTS}

The experiments and both simulations present qualitatively similar images (Figs. 1, 2) and consistent results for the yield drag force, always directed downstream.

There are a priori four lengths in this problem: the channel width $w_{c}$, the obstacle diameter $d_{0}$, the bubble size $\sqrt{A}$; and the cut-off length $L_{c}$. As far as we can tell, it is safe to assume that the channel length (if long enough) is irrelevant here. These four lengths can be reduced to three dimensionless parameters. We present the results using: $d_{0} / w_{c}$ which characterises the flow geometry; $d_{0} / \sqrt{A}$ which describes the foam-obstacle interaction; and $L_{c}^{2} / A$ which characterises the threshold for $\mathrm{T} 1$ rearrangements, and corresponds to the liquid fraction $\Phi$.

\section{A. Effect of obstacle to channel size ratio}

Potts model simulations indicate that the network yield drag is independent of the ratio of obstacle size to channel width, $d_{0} / w_{c}$ (Fig. 5a). This ceases to be valid at small $d_{0}$, when the obstacle is comparable in size to a bubble, and at large $d_{0}$, when the distance between the obstacle and the channel side is small [26].

The lack of dependence on $d_{0} / w_{c}$ that we find characterises the yielding behaviour of the foam: it means that only a small region near the obstacle is affected by the flow 16]. Nonetheless, the zone where the obstacle influences the flow is larger in 2D [34] than in 3D [16], as elastic or hydrodynamic interactions would suggest.

\section{B. Effect of obstacle to bubble size ratio}

Potts model simulations indicate that the network yield drag increases linearly with the obstacle size $d_{0}$ (Fig. 50) at fixed bubble area. This is consistent with the force increasing as $d_{0} / \sqrt{A}$, also suggested by the available Surface Evolver data, as well as by experimen-

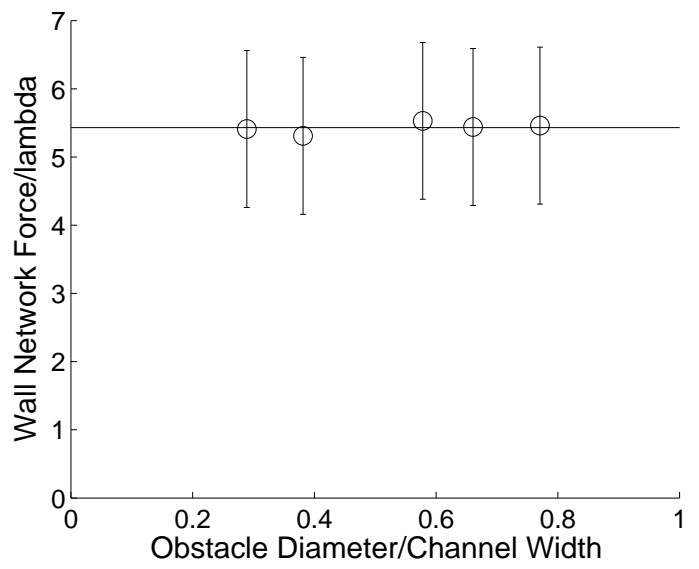

(a)

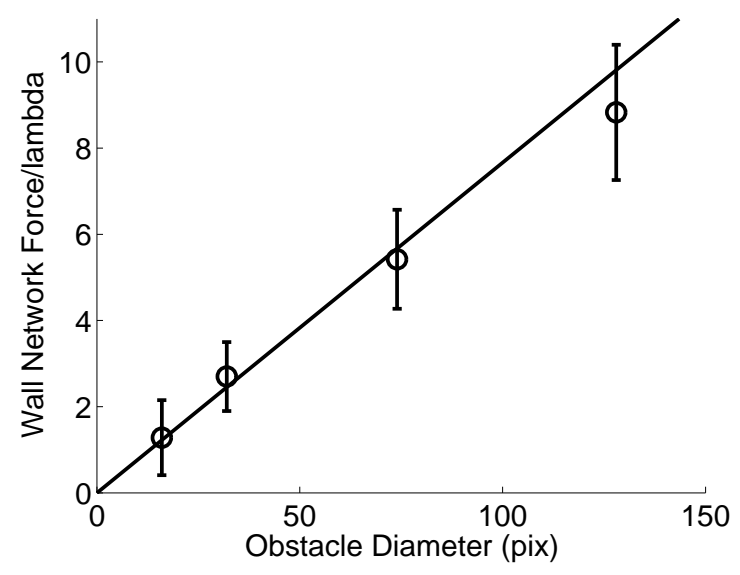

(b)

FIG. 5: Network contribution to the yield $\operatorname{drag} F_{Y}^{n}$ (expressed in unit of $\lambda$ ), measured in Potts model simulations with $A=100 \operatorname{pix}^{2}(\Phi=0.005)$. (a) $F_{Y}^{n}$ versus $w_{c}$, for $d_{0}=74$ pix; the solid line is the average (value 5.43). (b) $F_{Y}^{n}$ versus $d_{0}$, for $w_{c}=256$ pix; the solid line is a linear fit with zero intercept, $F_{Y}^{n}=0.77 d_{0} / \sqrt{A}$.

of the obstacle's spanwise dimension ("leading edge") [9]. Note that most elastic properties of a foam scale like $1 / \sqrt{A}[3]$. In fact, when $A$ increases, the density of bubbles and of bubble walls decreases, and so does a foam's elastic modulus (it would eventually vanish if there were only one large bubble left).

\section{Effect of liquid fraction}

We need to separate the effects of foam geometry, 


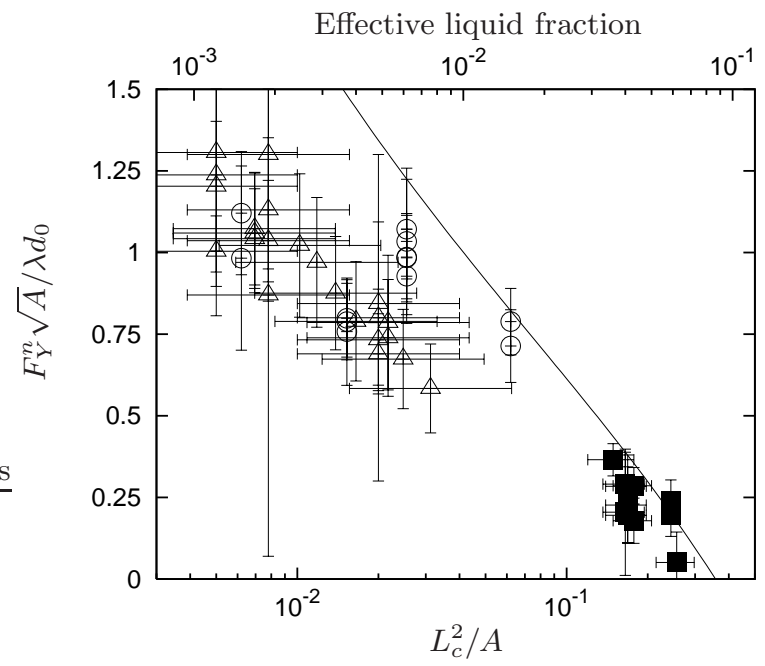

FIG. 6: The network contribution to the yield $\operatorname{drag} F_{Y}^{n}$, rescaled by $\lambda d_{0} / \sqrt{A}$, is plotted versus the dimensionless quantity $L_{c}^{2} / A$ (bottom scale), that is versus the effective liquid fraction $\Phi$ (top scale), in the range $10^{-3}<\Phi<10^{-1}$. All control parameters $\left(d_{0}, w_{c}, A\right.$ and $\left.\Phi\right)$ are varied. Vertical bars indicate the standard deviation of the force fluctuations in time around the plateau value. Horizontal bars indicate the uncertainty discussed in Sec. IV Data are from experiments (ם), Surface Evolver (o) and Potts model $(\triangle)$; the solid line denotes the analytical model, from eq. (B10), without adjustable parameters. Note that the horizontal scales are logarithmic and shifted with respect to each other.

rescale the network contribution to the yield $\operatorname{drag} F_{Y}^{n}$ by $d_{0} / \sqrt{A}$, and plot all our data as a function of $\Phi$. All the data, from both experiments and simulations, are well rescaled in the range $10^{-3}<\Phi<10^{-1}$ (Fig. 6). This is the main result of the present paper.

\section{DISCUSSION}

\section{A. Model}

The effect of the liquid fraction on the network drag can be understood as follows. A bubble of area $A$ detaches from the obstacle when its width is of order $L_{c}$, and thus its length is of order $A / L_{c}$. When $\Phi$ decreases, $L_{c}$ decreases too. Bubbles stretch more downstream, ber of bubble walls pulling the obstacle downstream increases; simultaneously, the number of walls upstream decreases. This larger up/downstream asymmetry results in an increase in the resulting drag $F_{Y}^{n}$. The contribution from the network (or bubble walls) increases as their number per unit length along the obstacle boundary, namely $L_{c}^{-1}$, and thus scales like $1 / \sqrt{\Phi}$.

However, the length of the region on which stretched bubbles act decreases, and the divergence in $1 / \sqrt{\Phi}$ is in fact softened by a geometrical factor. As shown in Appendix $\mathrm{B}$, we can estimate this factor by integrating the bubble wall contribution around the obstacle. When $\Phi$ increases, $F_{Y}^{n}$ decreases; it vanishes for $\Phi=0.086$. This is close to the rigidity loss value (eq. 8). Eq. (B10) is plotted in Fig. (6), without adjustable parameters. It shows qualitative agreement with the data over two decades of liquid fraction, suggesting that it captures the essence of the physics.

\section{B. Influence of the control parameters}

In the limit of low $\Phi$, the development of the above argument indicates that, provided that the obstacle diameter and the obstacle-wall distances are larger than the bubble diameter, $F_{Y}^{n}$ increases according to:

$$
F_{Y}^{n}=\frac{0.516}{\Phi^{1 / 4}} \frac{\lambda d_{0}}{\sqrt{A}}
$$

In simulations, if we multiply the bubble and obstacle diameters, expressed in units of the cut-off length, by the same prefactor, the network drag changes (data not shown), due to the change in $\Phi$.

Conversely, increasing only the bubble area $A$ at fixed $L_{c}$ simultaneously decreases both $d_{0} / \sqrt{A}$ and $\Phi$. This has two opposing effects, the former decreasing $F_{Y}^{n}$, the latter increasing it, resulting in an almost constant $F_{Y}^{n}$ (Fig. 7). (In fact there is a weak dependence on area, varying as $A^{-1 / 4}$.) This shows that the relevant way to vary the liquid fraction in simulations is to modify 


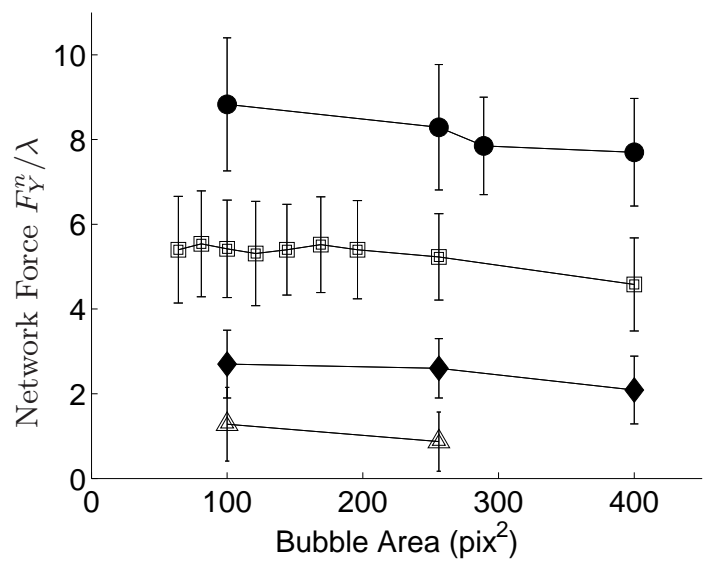

FIG. 7: Opposite effects in simulations of $F_{Y}^{n}$ (here with Potts model). When $A / L_{c}^{2}$ increases, both $d_{0} / \sqrt{A}$ and $\Phi \approx$ $A^{-1}$ decrease; so that $F_{Y}^{n}$ barely varies (eq. 10). Obstacle diameter $d_{0}$ equal to $16(\triangle), 32(\diamond), 74(\square)$ and $128(\bullet)$.
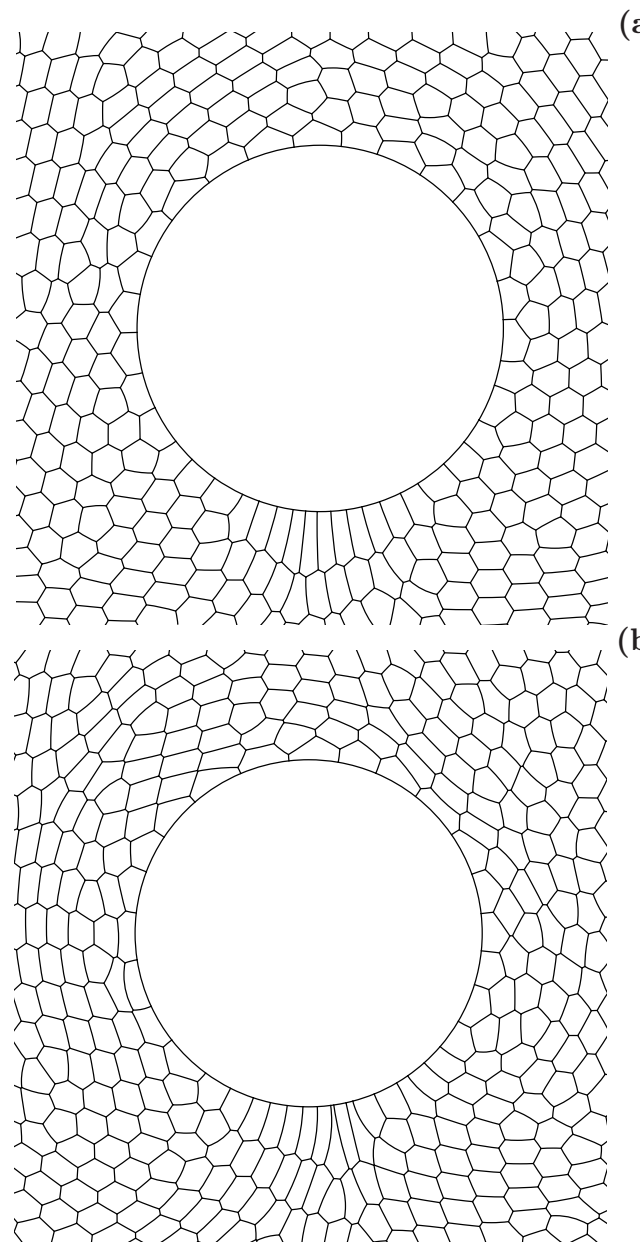

(b)

FIG. 8: Surface Evolver simulation of a very dry foam: zoom around the obstacle. (a) $\Phi=3.710^{-3}$; (b) $\Phi=$ $610^{-6}$. Here $d_{0}=4.8 \mathrm{~cm}, A=0.16 \mathrm{~cm}^{2}, w_{c}=10 \mathrm{~cm}$. Flow from top to bottom.

\section{Saturation at low $\Phi$}

Surface Evolver simulations allow us to probe the range $10^{-5}<\Phi<10^{-3}$. They indicate that the force saturates below $\Phi \sim 10^{-3}$, in agreement with our preliminary experiments of a foam confined between glass plates (data not shown).

Direct observation of simulation images of very dry foams (Fig. 8) confirms that the up/downstream asymmetry in the number of bubbles touching the obstacle is around 10, roughly independent of liquid fraction.

The model seems to correctly describe the squashing and stretching of bubble shapes. However, the interpolation between both extreme values assumes a phenomenological expression (eq. B4). It seems approximately valid only for $10^{-3}<\Phi<10^{-1}$ (see Appendix B). It applies to other obstacle shapes, such as an ellipse [35].

\section{Yield drag versus yield stress}

Princen and Kiss [36] have shown that in three dimensions a foam's yield stress scales as: $\sigma_{Y}=\gamma(1-$ $\left.\Phi_{3 D}\right)^{1 / 3} Y\left(\Phi_{3 D}\right) / R_{32}$, where $R_{32}$ is the surface-volume mean radius (Sauter radius), and $Y\left(\Phi_{3 D}\right)$ a decreasing function of $\Phi_{3 D}$ which is approximately $Y\left(\Phi_{3 D}\right) \simeq$ $-0.080-0.114 \ln \Phi_{3 D}[36]$.

At this stage, it is worth discussing the fundamental differences between yield stress and yield drag.

The yield stress or yield strain is an intrinsic property of the foam. On the other hand, the yield drag depends on the geometry of the flow: the foam does not yield everywhere around the obstacle, and especially not at angles $|\theta| \approx \pi / 4$ from the downstream direction, as appears both in experiments (Fig. 1) and in simulations (Figs. 2 and 8). This spatial dependence implies that the relation between yield stress and yield drag is nontrivial and $\Phi$-dependent. In particular, in a dryer foam (Fig. 8), the region where bubbles reach their maximal deformation is narrower [34]. 
only shear, but also elongation, especially near the front and back of the obstacle. When $\Phi$ decreases, the bubble elongation can become arbitrary large and dominates the contribution to the yield drag.

\section{CONCLUSION}

To summarise, we investigate the two-dimensional flow of a foam around a circular obstacle, within a long channel. Our deterministic (Surface Evolver) and stochastic (Potts model) simulations, as well as our model and experiments, complement and validate each other.

The yield drag is defined as the low-velocity limit of the interaction force between an obstacle and a flowing foam. The network contribution scales as the ratio of obstacle to bubble diameter, as long as this ratio is larger than unity, and is almost independent of the channel width. It increases (because more and more stretched bubbles accumulate behind the obstacle) as a power law when the liquid fraction contained in the foam decreases to $10^{-3}$, then saturates.

Having found a relevant definition of the liquid fraction, which is appropriate for experiments, simulations and theory, the dependence of yield drag with liquid fraction is well characterized. It is very different from that of local intrinsic properties such as the yield stress or shear modulus. This observation suggests that it will be difficult to deduce one quantity from the other. This should be kept in mind in future simulations, and has to be taken into account when modelling the foam behaviour.

Note that this definition of liquid fraction can be extended to other $2 \mathrm{D}$ flows in experiments (quasi-2D foam set-ups [19]) or simulations. Extension to 3D [15, 16] should also be simple, especially since the main effect of quasi-2D set-ups - external friction on the glass plate 37, 38] - does not seem dominant here. Our present effective liquid fraction based on rheological properties (T1s) facilitates the comparison between $2 \mathrm{D}$ and $3 \mathrm{D}$ fraction of water [12]). In simulations too, our definition immediately extends to 3D for both Surface Evolver, which uses as input parameter the cut-off area for a face which undergoes a T1; and Potts model, where the voxel (3D pixel) size plays the same role.

\section{Acknowledgements}

We gratefully acknowledge the help of Steven Thomas with Potts model simulations. We thank Isabelle Cantat for stimulating discussions, and emphasising the differences between yield drag and yield stress. CR thanks LANL and SC thanks LSP for hospitality. YJ is supported by US DOE under contract No. DE-AC52-06NA25396.SC is supported by EPSRC (EP/D071127/1) and his visit to Grenoble was supported by the Ulysses exchange programme. Part of this work was performed during the FRIT workshop [13].

\section{APPENDIX A: FORCE MEASUREMENTS IN EXPERIMENTS}

\section{Variation with foam thickness}

We present here new data concerning the drag exerted by a flowing foam of bubble area $A=16.0 \mathrm{~mm}^{2}$ on a circular obstacle of diameter $d_{0}=3 \mathrm{~cm}$. We measured the drag, as explained in full detail in [9], versus the foam velocity $V$ for six different foam thicknesses (Fig. 9a). As usual, the drag increases with increasing foam velocity.

More importantly for this paper, at given velocity, and especially at the limit of vanishing velocity, the drag increases with increasing foam thickness. This is due to (i) the decrease of liquid fraction with increasing foam thickness, as shown by the snapshots of the two extreme foam thicknesses in Fig. (97); (ii) the increase in the height of the films with increasing foam thickness. We fit the data by the formula $F=F_{Y}^{t}+A V^{a}$ to get the 

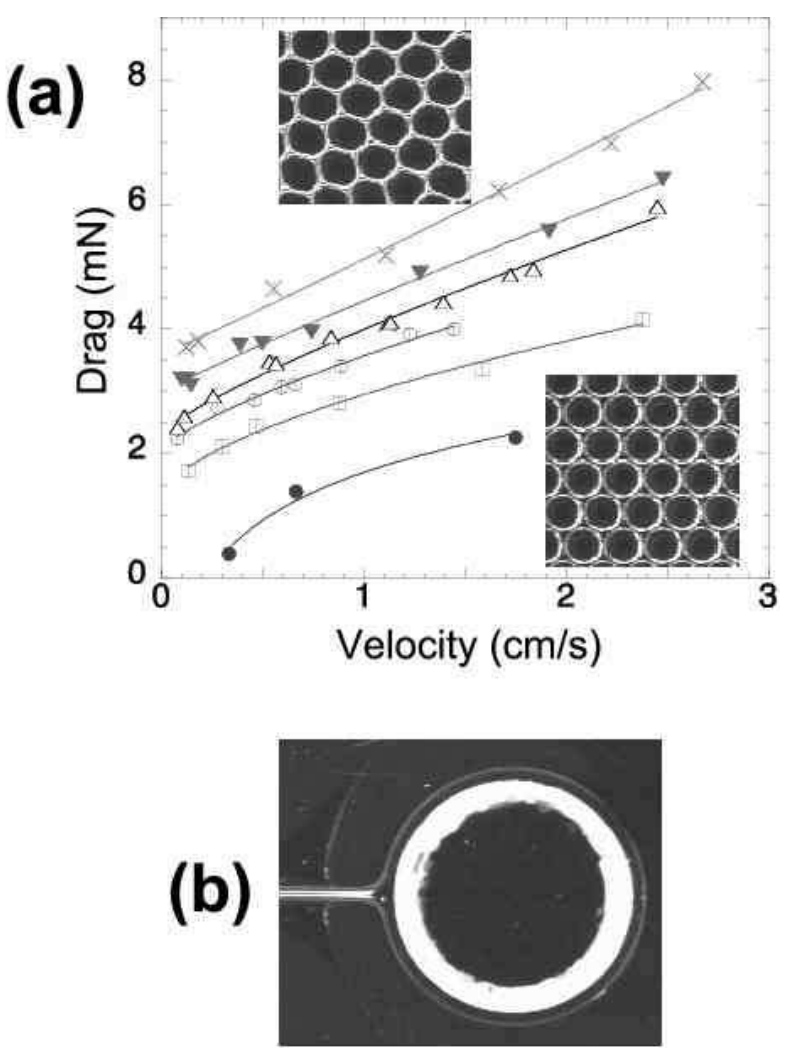

FIG. 9: (a) Drag versus velocity for various distances $h$ between the top plate and the bottom solution: $2.0(\bullet), 2.5$ $(\square), 3.0(\circ), 3.5(\triangle), 4.0(\mathbf{\nabla})$ and $4.5 \mathrm{~mm}(\times)$. Here $A=16.0$ $\mathrm{mm}^{2}$ and $d_{0}=3 \mathrm{~cm}$. The curves are the best fits by the formula $F=F_{Y}^{t}+A \times V^{a}$. Snapshots of the foam of the smallest (bottom) and highest (top) $h$ are also displayed. (b) Photograph of a single soap film pulling on the left side of the circular obstacle.

\section{thicknesses.}

Notably, for the smallest foam thickness $(h=2.0$ $\mathrm{mm}$ ) the foam is almost decompacted and the drag tends to vanish at low velocity. More precisely, the fit gives an unphysical negative value. This suggests that the rigidity loss transition [32] occurs for a foam thickness between 2.0 and $2.5 \mathrm{~mm}$.

\section{Comparison of network and total yield drags}

We measure the line tension directly as the force exerted on the obstacle by a single soap film, as shown in Fig. (9b), for two thicknesses. Its value is $0.44 \mathrm{mN}$

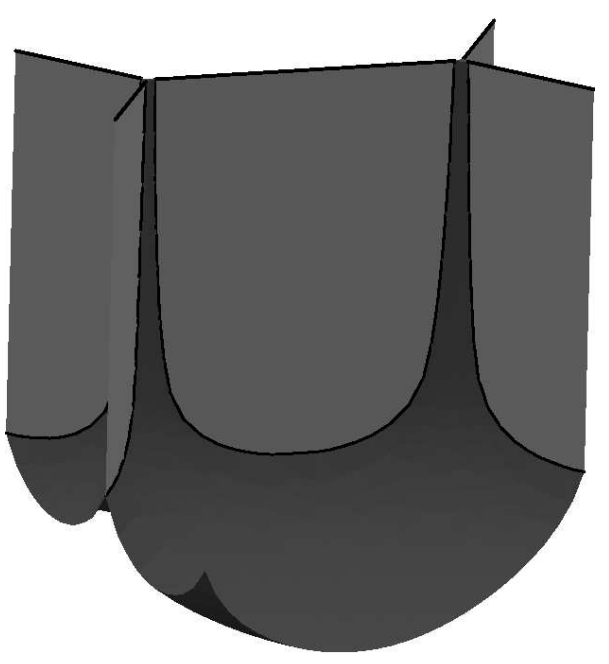

FIG. 10: Shape of the interfaces between bubbles, for bubbles of area $16.0 \mathrm{~mm}^{2}$ and volume $\mathcal{V}=16.0 \times 3.5 \mathrm{~mm}^{3}$, calculated with the Surface Evolver. Vertical films are shown in light grey and the liquid surface in dark grey. To reproduce the experiment, we enforce the hexagonal symmetry and include the buoyancy. For simplicity, the junction between lateral faces and the top plate is assumed to be orthogonal.

suggests that $\lambda / h \approx 110 \mathrm{mN} / \mathrm{m}$.

The actual gas-liquid interfaces have a 3D curvature to match tangentially the water surface and the glass plate (Fig. 10). This explains why the the measured value is between a lower bound, $\lambda / h=2 \gamma \approx 52$ $\mathrm{mN} / \mathrm{m}$ expected for a vertical soap film (that is, two flat gas/liquid interfaces), and an upper bound, $\lambda / h=$ $(2+\pi) \gamma \approx 134 \mathrm{mN} / \mathrm{m}$, expected for two films with circular cross section (see [39] for details).

Using the measured value of $\lambda$, we determine the absolute value of the network yield $\operatorname{drag} F_{Y}^{n}$ in experiments. We check (data not shown) that $F_{Y}^{n}$ is consistently of the same order of magnitude, but lower than, the value of $F_{Y}^{t}$ measured directly. The remaining part is attributed to the pressure contribution $F_{Y}^{p}$, to be described in a further paper [34]. The spatial variation of bubble height $h$ due to pressure differences is always less than $10 \%$, giving an upper limit to the spatial vari- 


\section{APPENDIX B: VARIATION OF $F_{Y}^{n}$ WITH THE CUT-OFF LENGTH}

We consider here only the bubbles touching the obstacle, and the contribution of their walls to the yield drag. We assume that (i) the foam is truly 2D and all bubbles have the same area (ideal 2D monodisperse foam); (ii) pressure $P$ is the same for each bubble, i.e. bubble walls are straight and all Plateau borders have the same radius of curvature $R$; (iii) the obstacle is much larger than the bubbles $\left(\sqrt{A} \ll d_{0}\right)$ so that we can neglect its curvature at the bubble scale. This latter approximation could in principle affect the bubbles upstream, which share a long edge with the obstacle. However, it should not greatly affect the bubbles downstream, which are the main contributors to the drag.

\section{Geometry}

To model a wet foam, we apply the decoration theorem 32]: the liquid is present only at the vertices which decorate an ideally dry foam. For a bubble touching the obstacle, we denote by $L$ the distance between two neighbouring vertices in contact with the obstacle (Fig. 117).

When the foam flows, bubbles attach to the obstacle upstream, and detach from it downstream. Visual observation of both experiments (Fig. 11) and simulations (Fig. 2) indicate that bubbles are flattened along the obstacle at the leading side of the obstacle, and that they progressively stretch streamwise at the trailing side.

$L$ reaches its minimum value downstream, where bubbles detach. There, two neighbouring (decorated) vertices come in contact, and $L$ equals the cut-off length $2 R$ (Fig. 11b).

On the other hand, for a new bubble to attach to the obstacle upstream, two bubbles must detach through the configuration of Fig. (11k). In this case, a vertex between three bubbles merges with one between two

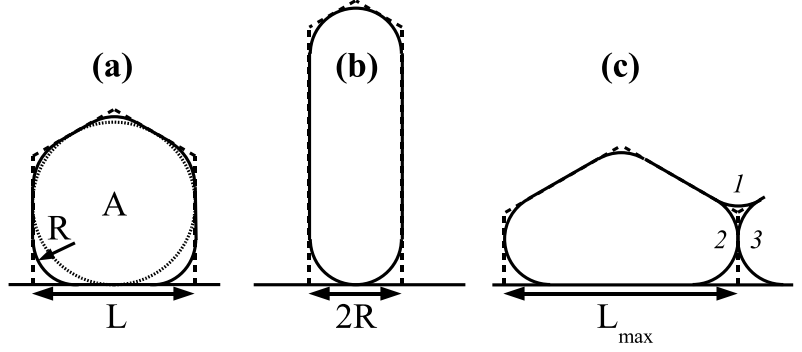

FIG. 11: Model configuration of the bubbles in contact with the obstacle. (a) Equilibrium configuration: the dashed line represents a polygonal bubble at $\Phi=0$, the dotted line is a circular bubble at $\Phi=\Phi_{c}$, and the plain line represents the intermediate case $\left(0<\Phi<\Phi_{c}\right)$, with straight edges and curved triangular vertices. (b) Configuration at the limit of detachment: two neighbouring vertices on the boundary of the obstacle come into contact. (c) Configuration at the point of attachment of a new bubble. There is one vertex between bubble 2, bubble 3 and the obstacle, and a second vertex between bubbles 1, 2 and 3. When these two vertices come in contact, bubble 1 attaches to the obstacle.

and now equals $(1+1 / \sqrt{3}) R$. This geometrically determines that the maximum bubble width $L_{\max }$ obeys:

$$
A=\left(1+\frac{1}{\sqrt{3}}\right) R L_{\max }+\frac{L_{\max }^{2}}{4 \sqrt{3}} .
$$

Inverting eq. (B1) yields $L_{\max }$ :

$$
\begin{aligned}
L_{\max }(A, R)= & 2 \sqrt{(\sqrt{3}+1)^{2} R^{2}+A \sqrt{3}} \\
& -2(\sqrt{3}+1) R .
\end{aligned}
$$

At low liquid fraction, $L_{\max }$ tends to a finite value, namely $\sqrt{4 A \sqrt{3}}$; there is no singularity at vanishing $R$. Conversely, at high liquid fraction, $L_{\max }$ varies greatly with $R$, so it is preferable to rewrite eq. (B2) and determine $R$ from the measurement of $L_{\max }$ :

$$
R\left(A, L_{\max }\right)=\left(1+\frac{1}{\sqrt{3}}\right)^{-1}\left(\frac{A}{L_{\max }}-\frac{L_{\max }}{4 \sqrt{3}}\right) .
$$

\section{Continuous assumption}

We assume that the shape of the bubbles varies smoothly from the configuration of Fig. (11k) upstream to that of Fig. (11) downstream: $2 R<L<L_{\max }$. 
switch from a discrete to a continuous description of the bubbles. We thus consider $L$ as a continuous function of the ortho-radial angle $\theta$ along the obstacle boundary: $\theta=0$ downstream, $\pi$ (and $-\pi$ ) upstream. Equivalently, $L^{-1}$ is the linear density of vertices along the obstacle boundary.

Then $L(0)=2 R, L( \pm \pi)=L_{\max }$. To interpolate between these values, we assume the following phenomenological dependence, reflecting that all bubbles in the range $|\theta| \geq \pi / 2$ appear squashed against the obstacle:

$$
\begin{aligned}
|\theta| \leq \pi / 2: \quad L(\theta)= & \left(R+\frac{1}{2} L_{\max }\right)+ \\
& \left(R-\frac{1}{2} L_{\max }\right) \cos 2 \theta \\
|\theta| \geq \pi / 2: \quad L(\theta)= & L_{\max } .
\end{aligned}
$$

Since each bubble edge exerts a pulling force of magnitude $\lambda$ along the outward normal vector of the obstacle boundary, the network contribution to the drag is

$$
F=\frac{\lambda d_{0}}{2} \int_{-\pi}^{\pi} \frac{\cos \theta}{L(\theta)} \mathrm{d} \theta
$$

To compute this integral, we introduce two dimensionless variables, both functions of $A$ and $R$ :

$$
\begin{aligned}
\varepsilon & =\frac{R}{\sqrt{A}}, \\
\beta & =\frac{L_{\max }}{2 R} .
\end{aligned}
$$

The physical meaning of $\varepsilon$ is equivalent to the liquid fraction, since

$$
\Phi=(2 \sqrt{3}-\pi) \varepsilon^{2}
$$

On the other hand, $\beta$ quantifies the amount of up/downstream asymmetry, that is, the squashing and stretching of bubbles. It increases when $\Phi$ (or equivalently $\varepsilon$ ) decreases (eq. B2):

$$
\beta(\Phi)=\sqrt{(\sqrt{3}+1)^{2}+\frac{(6-\sqrt{3} \pi)}{\Phi}}-(\sqrt{3}+1) .
$$

When $\Phi$ goes to zero, $\varepsilon$ goes to zero too, and $\beta$ diverges.

Using these variables, eq. (B5) yields

$$
F=\frac{\lambda d_{0}}{L_{\max }}\left[\frac{\beta}{\sqrt{\beta-1}} \arctan (\sqrt{\beta-1})-1\right] .
$$

At high liquid fraction, the force $F$ vanishes when $\beta=1$, that is (eq. B9) when:

$$
\Phi=\frac{2 \sqrt{3}-\pi}{2+\sqrt{3}}=0.086
$$

At low liquid fraction, we develop eq. B10 to leading order in $\beta$ and insert the leading order term of eq. (B9) to obtain eq. (10).
[1] R. G. Larson, The Structure and Rheology of Complex Fluids, Oxford University Press, New York (1999).

[2] A. Saint-Jalmes and D. J. Durian, J. Rheol. 43, 1411 (1999).

[3] D. Weaire and S. Hutzler, The physics of foams, Oxford University Press, Oxford (1999).

[4] R. Höhler and S. Cohen-Addad J. Phys.: Condens. Matt. 17, R1041 (2005).

[5] D.M.A. Buzza, C.-Y. D. Lu and M.E. Cates, J. Physique II 5, 37 (1995).

[6] F. Graner, Y. Jiang, E. Janiaud and C. Flament, Phys. Rev. E 63:11402 (2001).

[7] H.M. Princen, J. Coll. Int. Sci. 91, 160(1983).

[8] S.A. Khan and R.C. Armstrong, J. Non-Newtonian
[9] B. Dollet, F. Elias, C. Quilliet, C. Raufaste, M. Aubouy, and F. Graner, Phys. Rev. E 71:031403 (2005).

[10] P L J. Zitha, Transp. Porous Media 52, 1 (2003); S.J. Cox, S. Neethling, W.R. Rossen, W. Schleifenbaum, P. Schmidt-Wellenburg and J.J. Cilliers, Colloids Surf. A 245:143 (2004).

[11] I. Cantat, N. Kern and R. Delannay Europhys. Lett. 65: 726 (2004).

[12] N. D. Denkov, S. Tcholakova, K. Golemanov, V. Subramanian and A. Lips, Coll. Surf. A, 282-283:329 (2006).

[13] Workshop on Foam Rheology In Two dimensions (FRIT), Aberystwyth (UK), June 2005, http://users.aber.ac.uk/sxc/frit.html 
[15] S.J. Cox, M.D. Alonso, S. Hutzler and D. Weaire, in Eurofoam 2000, MIT Verlag, (Bremen, 2000), eds. P. Zitha, J. Banhart, G. Verbist, p. 282-289, Proc. 3rd Euroconf. on Foams, Emulsions and Applications, Delft (The Netherlands), June 2000.

[16] J.R. de Bruyn, Rheol. Acta 44:150 (2004).

[17] I. Cantat and O. Pitois, J. Phys.: Condens. Matter 17: S3455 (2005).

[18] I. Cantat and O. Pitois, Phys. Fluids 18:083302 (2006).

[19] M.F. Vaz and S.J. Cox, Phil. Mag. Letts. 85:415 (2005).

[20] E. Janiaud, D. Weaire and S. Hutzler, Coll. Surf. A. In press. (2007).

[21] This is due to the left-right symmetry of a bubble wall meeting the solid boundary. This is independent of the hydrophilic or hydrophobic nature of the solid surface (which would only play a role, through the presence or absence of a wetting film, in the energy dissipation, not relevant in the present paper).

[22] A. D. Gopal and D. J. Durian, Phys. Rev. Lett. 91: 188303 (2003).

[23] S. Cohen-Addad, R. Höhler and Y. Khidas, Phys. Rev. Lett. 93:028302 (2004).

[24] B. Dollet, F. Elias, C. Quilliet, A. Huillier, M. Aubouy, and F. Graner, Coll. Surf. A 263:101 (2005).

[25] K. Brakke. Exp. Math. 1:141 (1992).

[26] S.J. Cox, B. Dollet and F. Graner, Rheol. Acta 45:403 (2006).

[27] J. Lauridsen, M. Twardos and M. Dennin, Phys. Rev.
Lett. 89, 098303 (2002).

[28] M. Twardos and M. Dennin, Phys. Rev. E. 71: 061401 (2005).

[29] Y. Jiang, P. J. Swart, A. Saxena, M. Asipauskas and J. A. Glazier, Phys. Rev. E 59:5819 (1999).

[30] D.J. Srolovitz, M.P. Anderson, G.S. Grest and P.S. Sahni, Scripta Metall. 17241 (1983).

[31] E. A. Holm, J. A. Glazier, D. J. Srolovitz and G. S. Grest, Phys. Rev. A 43:2662 (1991).

[32] F. Bolton and D. Weaire, Phys. Rev. Lett. 65:3449 (1990).

[33] S.J. Cox, D. Weaire, and M.F. Vaz, Eur. Phys. J. E 7: 311 (2002).

[34] B. Dollet and F. Graner, to appear in J. Fluid. Mech. (2007), physics/0606170

[35] B. Dollet, M. Durth and F. Graner, Phys. Rev. E 73 061404 (2006).

[36] H. M. Princen and A. D. Kiss, J. Colloid Interface Sci. 128, 176 (1989).

[37] E. Janiaud, D. Weaire and S. Hutzler, Phys. Rev. Lett. 97:038302 (2006).

[38] Y. Wang, K. Krishan and M. Dennin, Phys. Rev. E 73: 031401 (2006).

[39] B. Dollet, Écoulement bidimensionnels de mousse autour d'obstacles, $\mathrm{PhD}$ thesis, unpublished, available on-line at http://www-lsp.ujf-grenoble.fr/pdf/theses/dtbn.pdf 

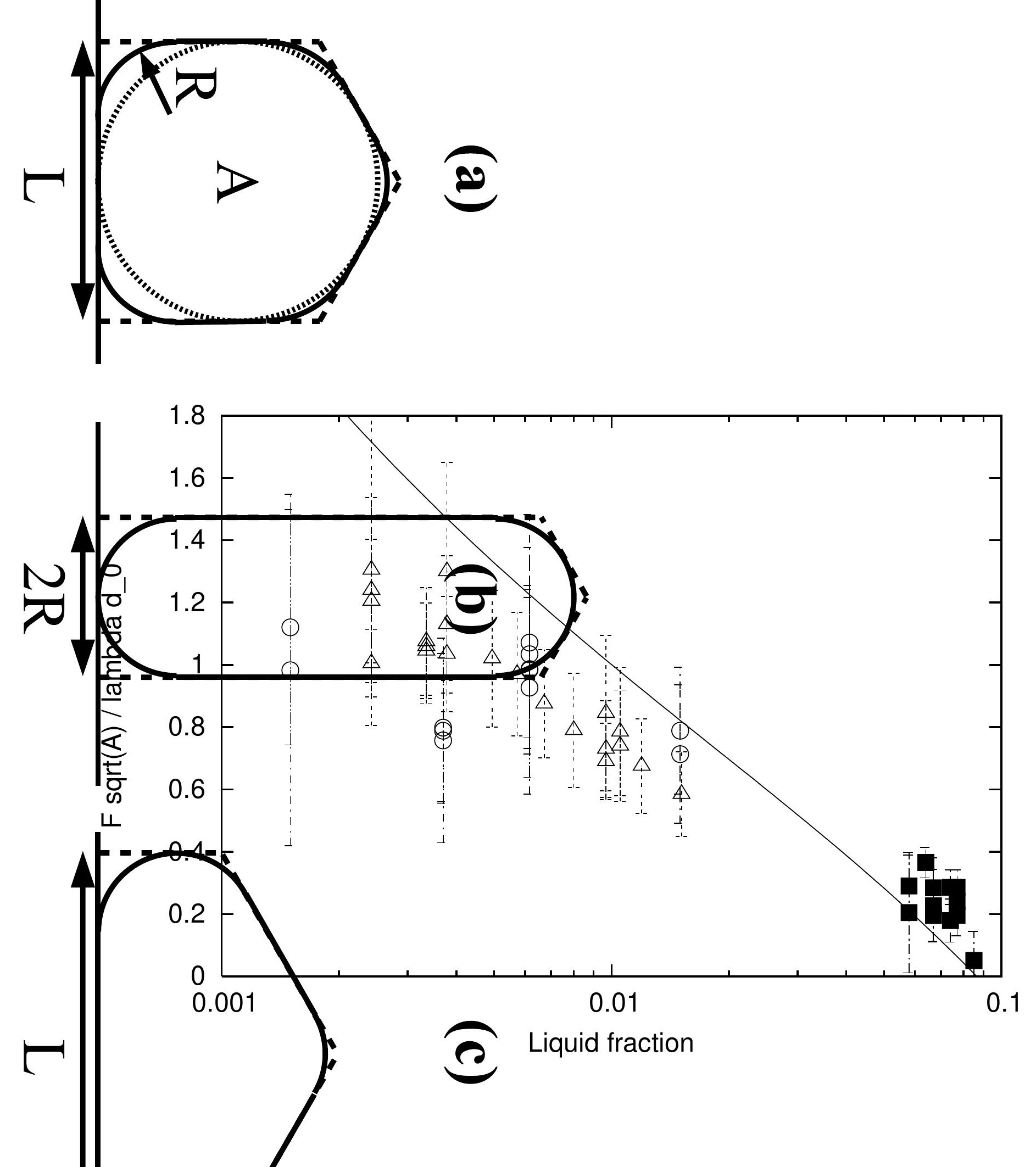


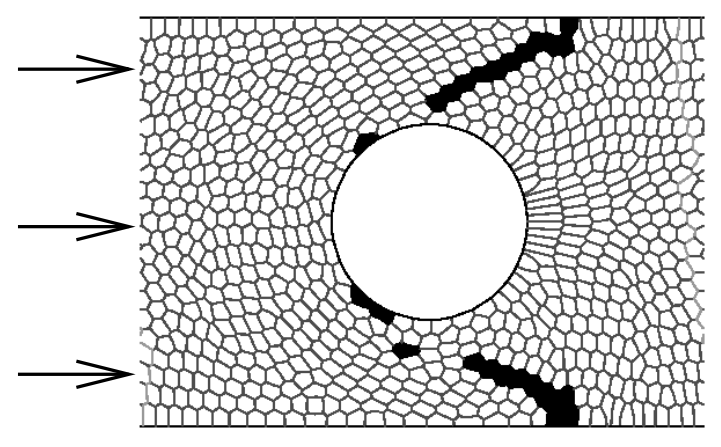

